\title{
Escalas de producción en economías mineras. El caso de Chile en su dimensión regional
}

Nathaly Rivera. Universidad Católica del Norte, Antofagasta, Chile.

Patricio Aroca. Universidad Adolfo Ibáñez, Viña del Mar, Chile.

RESUMEN | Una proporción importante de la producción de cobre en Chile está geográficamente concentrada en dos regiones: Antofagasta y Atacama. A pesar de la evolución positiva del precio del cobre, el ingreso promedio de estas localidades ha presentado variaciones. Así, durante periodos de incrementos en el precio del cobre el ingreso promedio de Atacama ha aumentado, mientras que lo mismo no ha ocurrido en Antofagasta. Este trabajo analiza esta diferencia a partir del rol de las escalas de producción en el sector minero. Utilizando un análisis de sensibilidad para un modelo insumo-producto desagregado, se calculan encadenamientos y multiplicadores por escalas de producción a nivel regional. Esta estrategia permite identificar y cuantificar el impacto de cada escala de producción, confirmando efectos heterogéneos de la minería sobre la producción e ingreso local, aspectos que adquieren especial relevancia al analizarse la política minera actual en Chile.

PALABRAS CLAVE | desarrollo regional y local, economía regional, política regional.

ABSTRACT | An important share of Chilean copper production is located in two regions: Antofagasta and Atacama. In recent years there has been a rise in the price of copper, and the incomes of the inhabitants of these regions have varied differently as a response to the variations in the price of copper. This article analyzes these differences between the evolution of regional income based on the size or scale of production in the mining sector. Using an input-output model disaggregated by the scale of production (large versus small and medium-sized scales) of the mining sector for each region, linkages and multipliers have been calculated and a sensitivity analysis carried out in order to test the strength of the estimations. This strategy allowed for identifying and calculating the impact of each scale on local income and production, showing the heterogeneous impact of the mining sector on local economies, which is very relevant for evaluating the current Chilean mining policy.

KEY WORDS | regional and local development, regional economy, regional policy.

Recibido el 20 de julio de 2012, aprobado el 9 de agosto de 2013

Email: Nathaly Rivera, narivera@ucn.cl | Patricio Aroca, patricio.aroca@uai.cl

El autor agradece el financiamiento parcial del núcleo de la Iniciativa Científica Milenio "Ciencia Regional y Políticas Públicas" del Ministerio de Economía, Fomento y Turismo; y del Centro de Estudios de Conflicto y Cohesión Social del programa CONICYT/ FONDAP/15130009, ambos de la República de Chile. 


\section{Introducción}

El objetivo de este trabajo es identificar y cuantificar el rol que cumplen las diferentes escalas de producción que caracterizan la minería del cobre, en el desarrollo de las regiones en Chile. Para ello se compara el caso de las principales regiones mineras en el país, Antofagasta y Atacama, cuya estructura productiva se diferencia principalmente por el porcentaje de participación regional de la pequeńa y mediana minería y por un dinamismo heterogéneo observado en el ingreso promedio de sus habitantes. Las condiciones geológicas de la región de Antofagasta han propiciado la instalación de un complejo productivo de gran escala en la zona, contrariamente a lo que ocurre con Atacama, donde parte importante de la producción regional es generada por empresas de pequeña escala. Esta situación, junto a la convicción de que en una región sustentable el ingreso de sus habitantes es maximizado (Capello, 2007), da lugar a la hipótesis que se contrastará en este trabajo: la producción de pequeña escala genera mayores impactos sobre el bienestar regional, debido a su tecnología de producción. Para contrastar esta hipótesis se utilizarán los niveles de ingreso per cápita como aproximación al bienestar regional.

A partir de las matrices insumo-producto regionales más actuales publicadas por el Instituto Nacional de Estadísticas para 1996 (INE, 2004), se calculan multiplicadores de la producción y del ingreso para ambas regiones, considerando una desagregación por tamaño de producción del sector Minería. Estos multiplicadores son obtenidos bajo los supuestos de sistemas abiertos y cerrados. Mientras que el supuesto de sistema abierto asume que la totalidad de los ingresos generados por los habitantes de la región son extraídos de ella, la consideración de un sistema cerrado asume que todos los ingresos son retenidos en la región. Estos supuestos, por construcción, funcionan como intervalos para el verdadero valor del efecto. Con esta estrategia de identificación se buscará evaluar el impacto que cada escala de producción posee a nivel regional, por potenciales efectos máximos y mínimos.

El siguiente trabajo se divide en cinco secciones. En la primera se presenta una revisión bibliográfica que, desde la economía regional, ayuda a entender los factores considerados en el análisis. La siguiente sección ofrece una descripción del desarrollo que la actividad minera ha tenido a escala regional en Chile. La tercera sección describe brevemente la metodología propuesta por Wolsky (1984) para la desagregación sectorial de las matrices insumo-producto, su análisis de sensibilidad para situaciones con información incompleta, y el cálculo de encadenamiento y multiplicadores. Luego se muestran los datos utilizados y los resultados obtenidos por región. Finalmente se presentan las conclusiones, destacando ejes de relevancia en la posible adopción de una estrategia que permita abordar el desarrollo de las regiones mineras.

\section{El rol de las escalas de producción en la minería}

El capital regional y la capacidad empresarial fueron destacados inicialmente por Chinitz (1961) como factores relevantes en la producción regional, al ser descritos como aspectos interindustriales que influyen en los costos de los factores de una empresa. Mientras que al capital se le ha atribuido un carácter móvil en el espacio, el 
autor observa que las empresas de tamaño pequeño prefieren realizar nuevas inversiones en negocios locales y no así en lugares externos a la unidad espacial, lo que permite diferenciar las pautas de crecimiento entre regiones ${ }^{1}$. Al mismo tiempo, el autor propone la reconsideración de la homogeneidad regional de la capacidad empresarial, al destacar que la función de utilidad de emprendimiento será más atractiva para aquellas industrias dominadas por pequeñas empresas, debido a las facilidades de entrada.

A partir del trabajo de Chinitz, las distintas escalas de producción de una industria han sido consideradas por la literatura como una pieza clave en las pautas de crecimiento económico de una región. La forma de organización de la industria dominante y su nivel de producción actúan como factores determinantes en el nivel de operación de una región, al influir en la cantidad de capital móvil y de vínculos interindustriales existentes (Heim, 1983), así como en la productividad, emprendimiento (Rosenthal \& Strange, 2003; 2010) y en los niveles de generación de empleo (Loveman \& Sengenberger, 1990; Spilling, 1998). Saxenian (1994, citado en Rosenthal \& Strange, 2003) se refiere a este punto coincidiendo con Chinitz al concluir que la productividad de un ambiente económico es mayor cuando la estructura industrial está dominada por pequeñas empresas, como consecuencia de la flexibilidad y del nivel de emprendimiento que las caracteriza.

\section{La relevancia de la pequeña minería}

La relevancia de las operaciones de la pequeña minería ha sido destacada en aspectos como reservas pequeñas de mineral $^{2}$, menores tiempos de implementación de sus operaciones e inversión inicial, menores requerimientos de infraestructura y mayores ratios de empleo por unidad de producción (Argall, 1978, citado en Ghose \& Roy, 2007). Empíricamente, el impacto socioeconómico de la pequeña y mediana minería ha sido analizado en países en desarrollo, destacándose la importancia de esta escala de producción en aspectos como la reducción de la pobreza, especialmente en zonas rurales (Hilson 2002a, 2002b; Yelpaala \& Ali, 2005), la contribución a las bases de minerales de exportación e ingresos de divisas (Shen \& Gunson, 2006), y la generación de empleos y habilidades empresariales (Ghose \& Roy, 2007), considerando así el impacto favorable que en términos socioeconómicos generan la pequeńa y mediana minería en las zonas en las cuales se localizan. Cuando la pequeña minería se localiza en regiones económicamente atrasadas, estimula el ingreso y su distribución, la creación de nuevos puestos de trabajo y un mejor aprovechamiento de los recursos internos, dando lugar a un rápido incremento en el estándar de vida de las personas.

$1 \longdiv { \text { Además de resaltar el carácter local en las inversiones de las pequeñas empresas, Chinitz se refiere } }$ al mayor requerimiento de insumos propio de este tipo de empresas, en comparación con aquellas de mayor tamaño, lo que generaría diferencias en la cantidad de interrelaciones productivas por escala de producción.

2 Este punto es profundizado por Hilson (2002a) y Ghose y Roy (2007), quienes resaltan la importancia de las empresas mineras de menor tamaño, financieramente viables en los depósitos minerales que son desechados por la gran minería, lo que denota un uso más eficiente de los recursos naturales de una región. 
No obstante el potencial de la pequeña minería, la industria minera se encuentra actualmente dominada por grandes empresas, siendo bastante menor el espacio a la producción de pequeña escala. Más aún, la gran minería del cobre en Chile se caracteriza por su alto porcentaje de propiedad extranjera, con la particularidad de exportar fuera de la región los beneficios generados en la localidad. Gunton (2003) se refiere a las potenciales distorsiones en la estructura económica de una región especializada en la extracción de recursos naturales cuando la industria minera se compone de grandes empresas extranjeras, debido a que estas impiden la emergencia de fuertes emprendimientos en la región. El autor coincide con las formulaciones de Chinitz (1961) y Saxenian (1994) sobre las barreras de entrada para pequeńas empresas en un ambiente cubierto por la producción de gran escala, refiriéndose a la fuga de los ingresos generados por este sector en forma de utilidades o dividendos a los propietarios extranjeros, particularidad que caracteriza a las grandes empresas en esta industria y no así a la minería de pequeña escala. Ghose y Roy (2007) denominan esta peculiaridad como el valor agregado de la pequeña minería, ya que el origen local de sus capitales puede generar una ganancia neta más grande para la comunidad. En términos empíricos, por ejemplo, Banks (1993) analizó la salida de utilidades que ha sufrido Papúa Nueva Guinea producto de la propiedad extranjera de sus empresas. El autor cuantificó la fuga de capitales en $70 \%$ del valor total de la producción minera para ese país.

Para el caso de Chile, en cambio, solo en Lufin (2002) se encuentra un breve comentario, donde el autor, en un análisis de la sustentabilidad de las regiones mineras, propone el aumento del valor agregado de las exportaciones, la retención de los excedentes de producción y el aumento del número de empresas de génesis local como modo de incrementar el desarrollo. Otros trabajos relacionados han concentrado la atención sobre la gran minería del cobre (GMC) y los impactos generados en la economía nacional o regional. En esta línea, Aroca $(2000 ; 2001)$ sugiere que la importancia de la GMC para la región de Antofagasta se da principalmente en términos de volumen de producción y no así en su capacidad para generar interrelaciones productivas ni en su aporte al ingreso o al empleo de la zona. A partir del cálculo de encadenamientos y multiplicadores, el autor estima que más de $75 \%$ del incremento adicional en la producción del sector no se traspasa en ingresos a los trabajadores de la región.

\section{Características generales de la minería en Chile}

\section{Distribución espacial de la minería del cobre}

La minería del cobre en Chile se concentra principalmente en la zona norte, especialmente en las regiones de Tarapacá, Antofagasta y Atacama. La producción conjunta de cobre fino en estas regiones supera el 75\% del total nacional. Este sector es considerado la principal actividad económica regional, con participaciones que van desde el 50\% hasta el 80\% del Producto Geográfico Bruto (PGB) ${ }^{3}$, y cuya primacía se mantiene constante desde las últimas dos décadas ${ }^{4}$.

3 La participación del sector minero en el 2010 para estas regiones fue de 53,8\% para la región de Tarapacá, 65,9\% para la región de Antofagasta, y 51,2\% para la región de Atacama (Banco Central de Chile, 2010).

4 Esto es cierto solo para las regiones de Antofagasta y Atacama. Para la región de Tarapacá, únicamente a partir de 1998 el sector minero comenzó a dominar la actividad económica regional, 
Sin embargo, las características del desarrollo local y los resultados derivados de la explotación de este sector primario-exportador son diferentes para cada región. Es en la región de Antofagasta donde se configura un ambiente adecuado para el desarrollo de un complejo productivo en torno a la actividad minera, a causa de sus condiciones geológicas y el liderazgo que mantiene en la producción nacional de cobre. Esto atrae un gran flujo de inversión extranjera directa a la zona, originando la explotación de grandes yacimientos mineros, responsables hoy del 53\% de la producción total de la GMC en Chile. Solo en la minería privada Antofagasta posee ocho de las quince grandes empresas que operan en el país, mientras que la minería estatal alcanza $60,4 \%$ de su producción en esta zona. Esto genera una diferenciación geográfica a partir de la concentración de yacimientos mineros de diferentes tamaños de producción.

Para mostrar lo anterior, en la Figura 1 se grafican las participaciones regionales en la producción nacional de cobre fino por grandes, medianas y pequeñas empresas para el período 2001-2008. La imagen da cuenta de la especialización a nivel nacional de la región de Antofagasta en la GMC, con 55\% en promedio de la producción nacional; y de la región de Atacama en la pequeña y mediana minería, con $45 \%$ en promedio para el mismo período. La región de Tarapacá no concentra especialización a nivel nacional por tamaño de producción ${ }^{5}$. La Tabla 1 confirma esta diferenciación geográfica en tamaños de producción, al presentar las participaciones por tamaño de empresa en la producción regional. A partir de esta tabla, es evidente la especialización regional de Tarapacá y Antofagasta en la GMC, mientras que en Atacama la pequeńa y mediana minería tienen una importante participación, como no ocurre en otra región, y que se vio acrecentada durante los períodos de alza del precio del cobre entre los años 2003 y 2005.

FIgURA 1 | Participación regional en producción nacional de cobre fino. Período 2001-2008

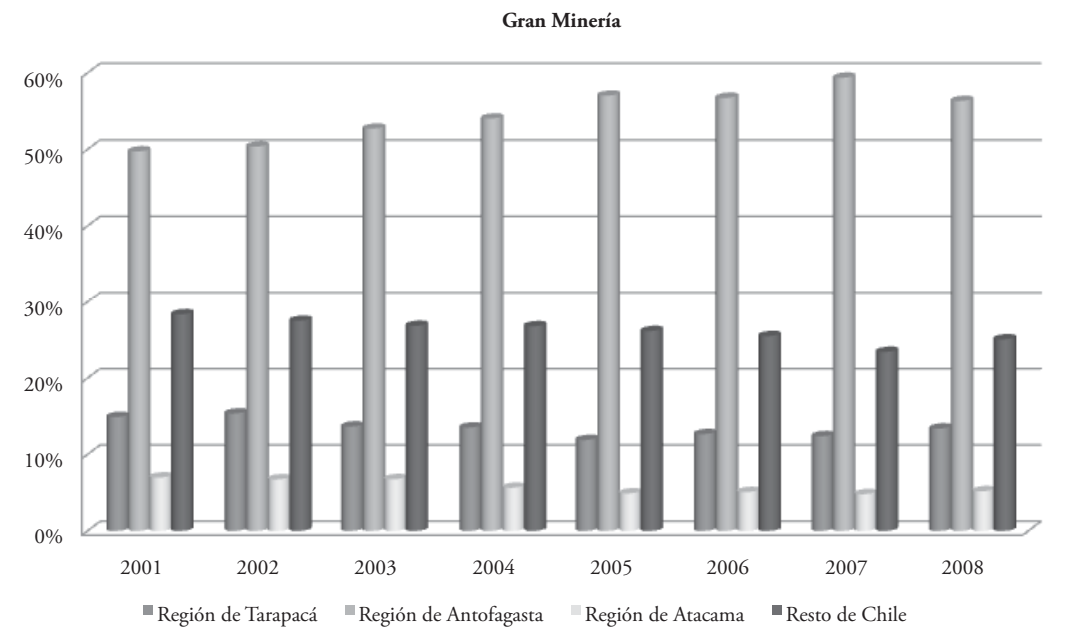

con una participación del 35\% del PGB de la zona.

5 A esto se suma el escaso desarrollo que la pequeña y mediana minería han tenido en la región, habiendo llegado a agotarse completamente durante los últimos ańos. 


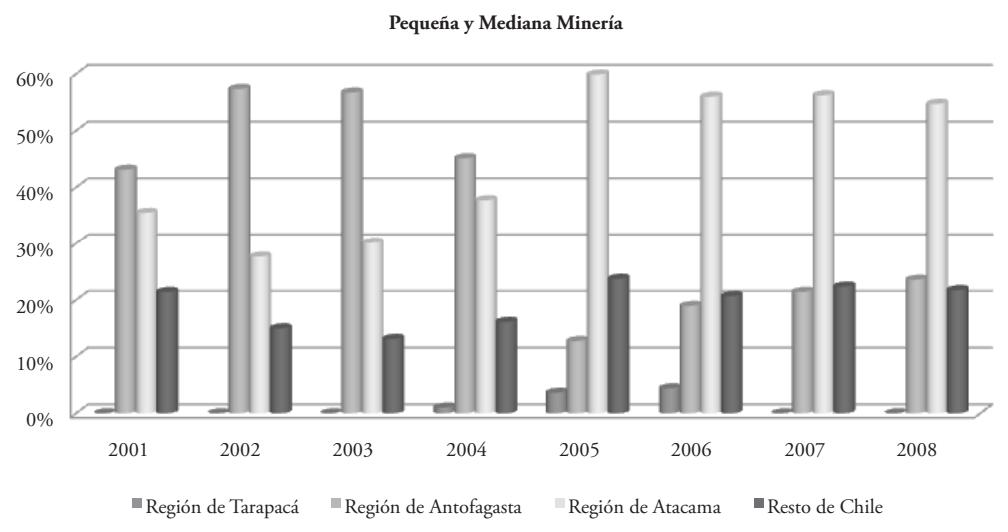

FUENTE ELABORACIÓN PROPIA SOBRE LA BASE DE DATOS DE LA COMISIÓN CHILENA DEL COBRE

TABLA 1 Participación por tipo de empresa, en la producción regional. Período 2007-2008

\begin{tabular}{|l|c|c|c|}
\hline $\begin{array}{c}\text { ESCALA DE } \\
\text { PRODUCCIÓN/ } \\
\text { REGIón }\end{array}$ & PEQUEÑA MINERÍA & MEDIANA MINERÍA & GRAN MINERÍA \\
\hline I Región & $0 \%$ & $0 \%$ & $100 \%$ \\
\hline II Región & $0,4 \%$ & $2,2 \%$ & $97,3 \%$ \\
\hline III Región & $11,6 \%$ & $32,3 \%$ & $56 \%$ \\
\hline
\end{tabular}

FUENTE COMISIÓN CHILENA DEL COBRE (2OIO)

La minería del cobre, sus escalas de producción y la propiedad del capital El criterio utilizado para clasificar las empresas mineras en distintos tamaños difiere del utilizado por el resto de los sectores productivos. De acuerdo con la Sociedad Nacional de Minería, la definición de gran, mediana y pequeña minería se hace a partir del número de toneladas métricas (tm) explotadas por ańo (véase Tabla 2).

TABLA 2 Tamaño de empresas en el sector minero

\begin{tabular}{|l|l|c|}
\hline $\begin{array}{c}\text { TAMAÑo DE } \\
\text { PRODUCCIÓN }\end{array}$ & \multicolumn{1}{|c|}{ DEFINICIÓN } & $\begin{array}{c}\text { PARTICIPACIÓN EN LA } \\
\text { PRODUCCIÓN NACIONAL }\end{array}$ \\
\hline Gran Minería & +3.000 .000 tm/año & $93 \%$ \\
\hline Mediana Minería & Entre 100.000 tm/año y 3.000 .000 tm/año & $5 \%$ \\
\hline Pequeña Minería & Menos de 100.000 tm/año & $2 \%$ \\
\hline
\end{tabular}

FUENTE SOCIEDAD NACIONAL DE MINERÍA (2009); COMISIÓN CHILENA DEL COBRE (2008)

La importancia de la distinción por tamaño de producción radica en una serie de factores diferenciadores que caracterizan a estas empresas. A la concentración geográfica de su explotación es posible agregar las diferencias en términos de productividad, donde la GMC alcanza niveles de eficiencia de mano de obra muy por sobre los niveles obtenidos por la pequeña y mediana minería, producto de la alta intensidad 
de uso del factor capital en su proceso de explotación ${ }^{6}$. Así, la producción de la GMC es intensiva en capital, mientras que la producción de la pequeña y mediana minería lo es en mano de obra. Otro factor diferenciador es la orientación al mercado de estas empresas. El mercado objetivo de la GMC es el extranjero, mientras que el mercado de la pequeña y mediana minería es mayoritariamente interno, con el poder comprador local representado por la Empresa Nacional de Minería (Enami).

Otro aspecto distintivo de los diferentes tamaños de producción es la estructura de propiedad del capital. La GMC se caracteriza por un capital de origen externo (estatal o extranjero), mientras que la pequeña y mediana minería nacen principalmente de iniciativas de grupos empresariales chilenos o dueños individuales locales (Sociedad Nacional de Minería, 2009; Comisión Chilena del Cobre, 2008). Este hecho genera un escenario diferente para las utilidades derivadas de la operación de estas empresas, ya que las ganancias que obtiene la pequeña minería pueden recircular en la región, vía consumo o inversión, mientras que las ganancias derivadas de la gran empresa en su mayoría son extraídas del territorio. A esto se agrega la alta identificación que poseen las empresas de tamaño menor con las comunidades locales donde están insertas sus faenas, y cuya causalidad es atribuida por Salas (2009a; 2009b) al origen de estas empresas.

\section{El impacto en ingresos}

La evolución en los precios de los commodities permite inferir algunas de las principales variables macroeconómicas de economías centradas en la explotación de recursos naturales. La Figura 2 muestra la evolución del precio nominal de la libra de cobre refinado, respecto de ello los períodos 2003 y 2006 muestran evidencias significativas para sugerir un cambio estructural en la tendencia ${ }^{7}$.

Debido a las ventajas que constantemente ha mostrado la variable Producto Interno en la literatura al momento de capturar la totalidad de la actividad económica, la Figura 3 muestra una comparación entre el PGB per cápita (eje vertical) y el Ingreso Promedio del Trabajo (eje horizontal) para Antofagasta y Atacama durante 1987-2009, utilizando como índice 100 a la región Metropolitana. En esta figura se aprecian los altos niveles de producto interno per cápita que exhibe Antofagasta, variable que alcanza valores muy por encima de los alcanzados por las regiones Metropolitana y de Atacama. Con altas tasas de participación de la GMC, Antofagasta es la región con mayor producción bruta por habitante a nivel país, habiendo alcanzado su punto más alto en 1990 con un índice de 202,21. Respecto del ingreso promedio derivado de la ocupación principal, este fue mayor en Antofagasta que en la región Metropolitana solo en tres períodos: 1990, 1996 y 2000, mientras que los ingresos de Atacama se han mantenido por debajo tanto de la Región Metropolitana como de su par minera, siendo su punto más bajo el 2003, con un índice de 58,05 respecto de la Región Metropolitana.

6 A modo de ejemplo y para el 2008, la productividad de la GMC alcanzó 147 tm/año de cobre fino por trabajador, mientras que en la pequeña minería este indicador llegó a 9 tm/año.

7 Estimaciones de cambio estructural con un test de Chow indican un cambio significativo al $1 \%$ en la tendencia observada del precio del cobre para los períodos 2003 y 2006. 
FIgura 2 | Precio nominal del cobre refinado. Período 1986-2010

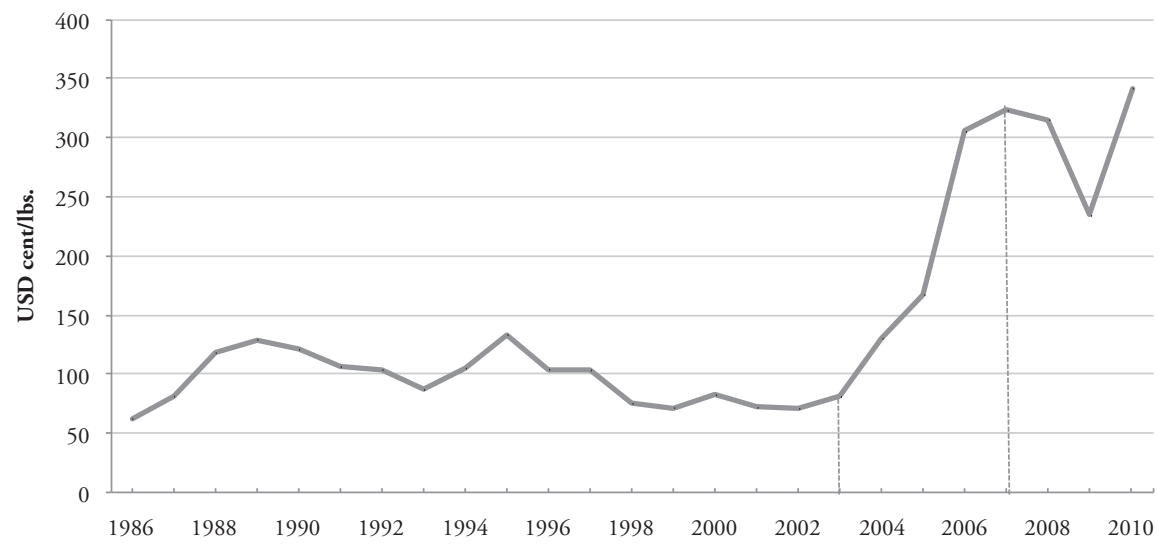

FUENTE COMISIÓN CHILENA DEL COBRE (20IO)

FIGURA 3 | Ingreso promedio del trabajo vs PGB per cápita. Índice $100=\mathbf{R M}$

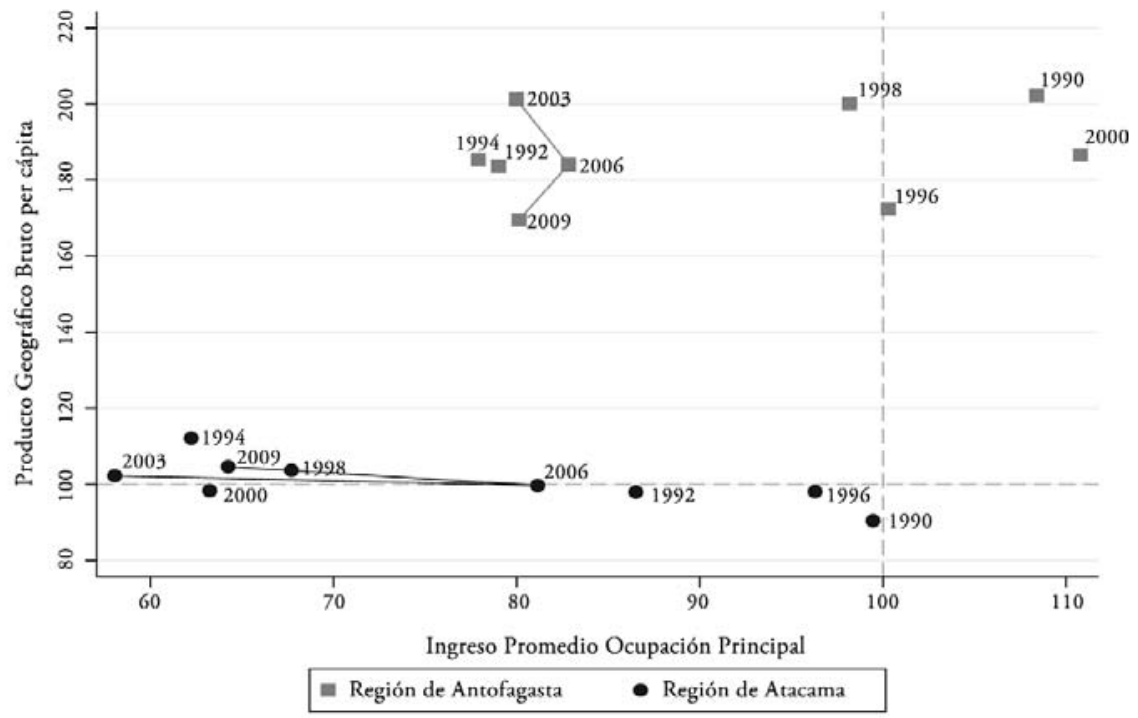

FUENTE BANCO CENTRAL DE CHILE Y ENCUESTA CASEN, VARIOS AÑOS

Para la Figura 3 es posible analizar lo ocurrido durante los períodos 2003, 2006 y 2009, durante los cuales el precio del cobre muestra un cambio significativo en su tendencia. De acuerdo con la Figura 2, el precio del cobre experimentó bajas sostenidas desde 1995 hasta 2003, y lo mismo ocurrió con el ingreso promedio en ambas regiones. Desde 2003, el cobre comienza su apreciación, alcanzando precios históricos en los ańos 2006 y 2007; sin embargo, luego de la crisis mundial, ocurrió una nueva caída en el precio del commodity. Para el período previo al 2007, el ingreso promedio regional de la ocupación principal presenta importantes alzas, sobre todo en Atacama, donde el incremento resultó cercano al 37,4\%, mientras que para Antofagasta fue solo de 1,77\%. El panorama poscrisis a partir de 2007, con bajas en el precio del cobre, fue perjudicial en términos de ingreso para ambas 
regiones, pero por sobre todo para Atacama, región en la que el ingreso promedio cayó a los niveles de principios de la década del 2000.

Contrario a lo esperado, la Figura 3 es categórica al mostrar la flexibilidad en el ingreso promedio de Atacama, cuando se la compara con Antofagasta. Dos posibles explicaciones surgen para entender la rigidez observada en el ingreso promedio de Antofagasta: un lento traspaso de beneficios derivados de las alzas en el precio del cobre a los salarios del sector minero en esta región; y una baja conectividad entre este sector y el resto de la actividad económica local, lo que impide el ajuste del ingreso promedio regional en términos globales.

Para dilucidar lo anterior, la Figura 4 muestra la evolución del PGB y el ingreso promedio exclusivamente para trabajadores del sector minero, durante los años 1996 a 2009. En esta figura es posible observar que el ingreso promedio de los trabajadores en el sector minería presenta una correlación positiva con la tendencia observada en el precio del cobre, sobre todo para el período 2006-2009. Esto significa que durante la baja en el precio del mineral, el ingreso de los trabajadores mineros disminuyó, mientras que durante el período de alza el ingreso se incrementó considerablemente. Este comportamiento consistente permitiría descartar, en un primer análisis, la posibilidad de que un lento ajuste de salarios sea la causa de la rigidez observada en las fluctuaciones de ingreso de Antofagasta y, más bien, deja entreabierta la posibilidad de que la baja conectividad interindustrial del sector sea la explicación más coherente para esta situación.

\section{FIGURA 4 Ingreso promedio del trabajo vs PGB - Sector Explotación de Minas y} Canteras

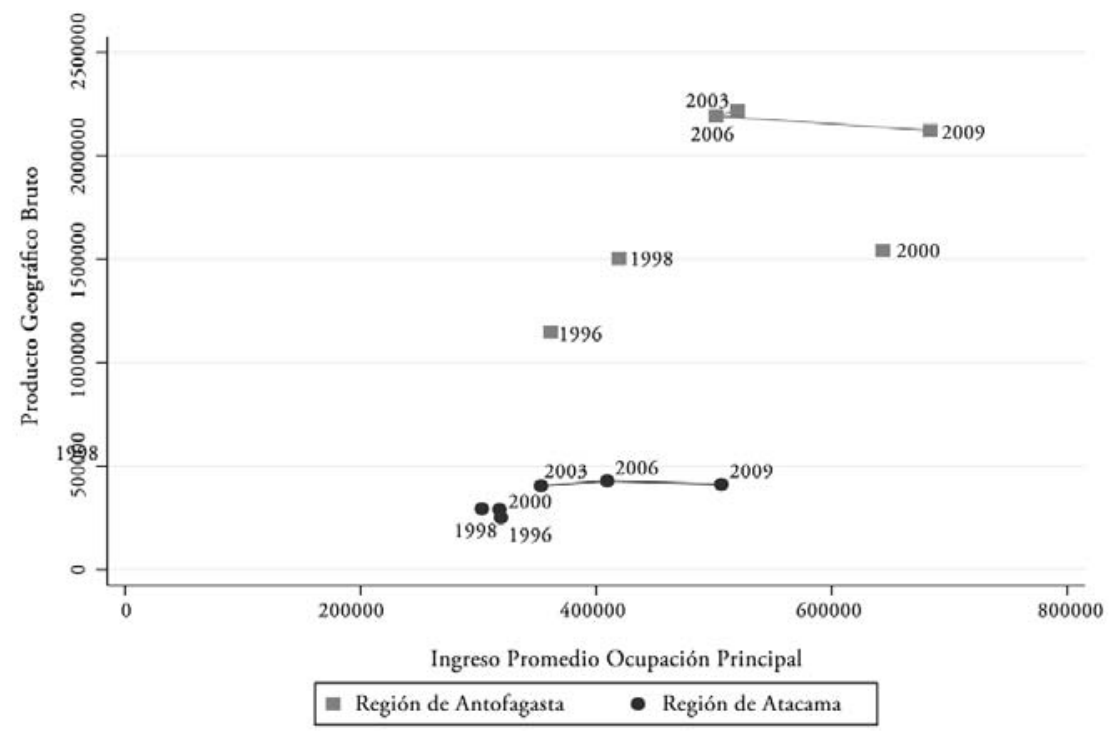

FUENTE BANCO CENTRAL DE CHILE Y ENCUESTA CASEN, VARIOS AÑoS

Para sustentar este planteamiento y observar el comportamiento técnico-productivo del sector minero en las regiones de análisis, la Figura 5 presenta las proporciones de ventas (abastecimiento) y compras (demanda) entre el sector minería y el resto 
de sectores. La comparación regional es útil al recordar los diferentes grados de participación de la pequeña y mediana minería en la producción de cobre fino de estas localidades.

La Figura 5 muestra que no existen mayores diferencias entre ambas regiones en las ventas que el sector minero realiza al resto de los sectores productivos. A causa de que este mineral se transa en mercados internacionales, la mayor actividad se produce al interior del mismo sector productivo. El mecanismo bajo el que operan los pequeños pirquineros nacionales, cuya extracción de cobre es vendida a la Empresa Nacional de Minería (Enami), provoca que las principales ventas del sector minero ocurran dentro del mismo sector productivo. La Figura 5 ilustra este patrón, que es incluso mayor en la región de Atacama, debido a la mayor presencia de pequeña minería.

FIgura 5 | Comportamiento técnico-productivo del Sector Minería. Período 1996
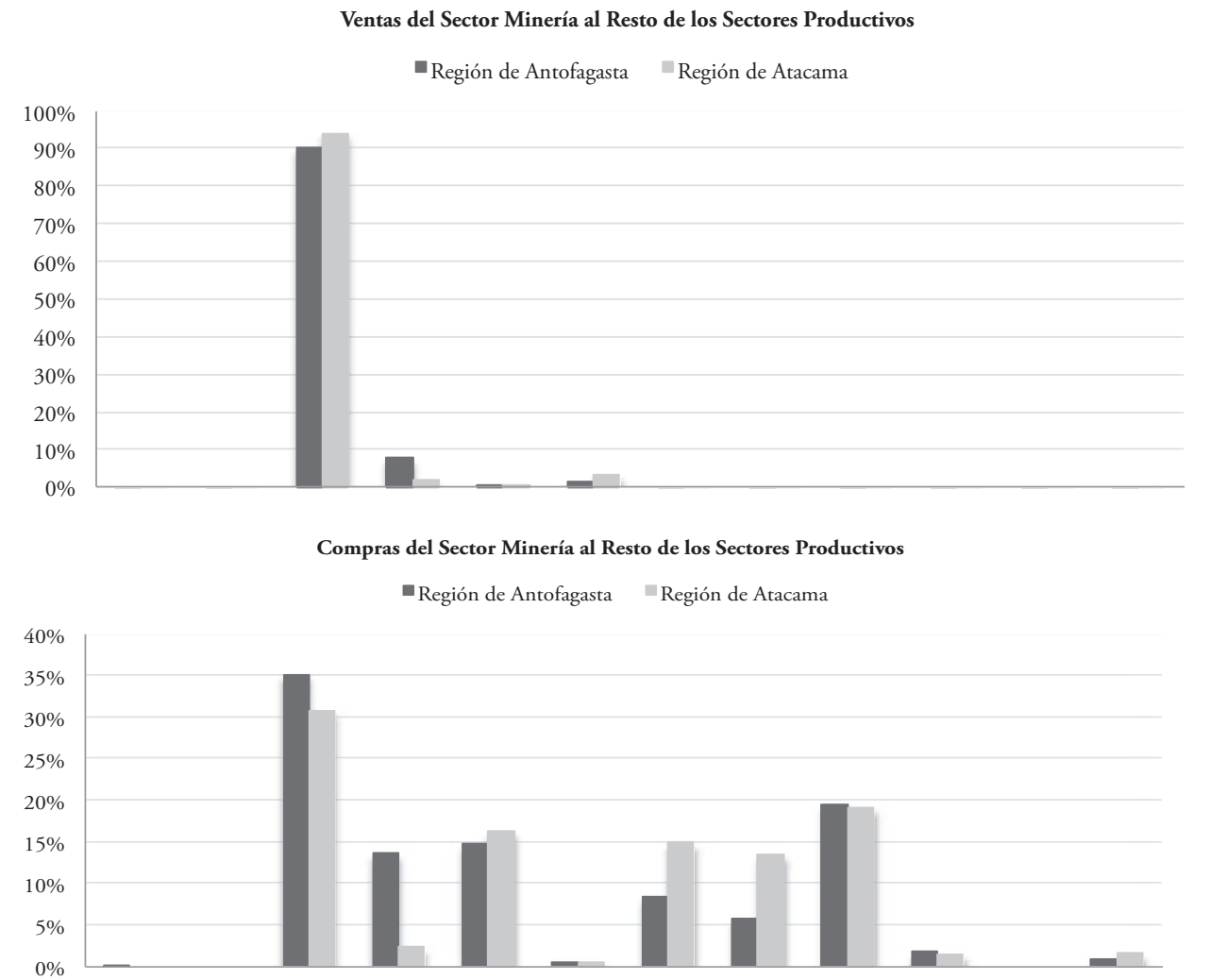

S01: Agropecuario Silvícola, S02: Pesca, S03: Minería, S04: Industria, S05: Electricidad Gas y Agua, S06: Construcción, S07: Comercio, Restaurantes y Hoteles, S08: Transporte y Comunicaciones, S09: Servicios Financieros y Empresariales, S10: Propiedad de Vivienda, S11: Administración Pública, S12: Servicios Sociales y Empresariales.

FUENTE MATRICES INSUMO-PRODUCTO REGIONALES. INE I 996

No obstante lo anterior, al analizar el patrón de compras del sector es clara la variabilidad que existe entre las regiones consideradas. En términos relativos, mientras la minería en Antofagasta demanda un mayor porcentaje de insumos desde el sector industrias, en la región de Atacama se observa una mayor demanda hacia los sectores 
de comercio, hoteles y restaurantes, y al sector transportes y comunicaciones. Una interesante explicación para esto es la integración vertical que caracteriza a la GMC, y que no ocurre en la pequeńa y mediana minería, esto genera una mayor autonomía en la GMC respecto del resto de sectores productivos en la región.

Al considerar las diferencias entre estas regiones surge de inmediato la posibilidad de que la estructura productiva del sector sea un aspecto clave en la disparidad en ingreso regional analizada previamente. La comparación descriptiva aquí analizada apoyaría esta posibilidad sugiriendo tres posibles causas: (i) las diferencias en volumen de producción entre la GMC y la pequeña y mediana minería; (ii) una menor integración vertical de la pequeña y mediana minería; (iii) la propiedad esencialmente local de la pequeña y mediana minería. Por otro lado, y con un ambiente propicio para la instalación de la pequeńa y mediana minería en Atacama, alzas en el precio del cobre mejoran el escenario para que pequeños pirquineros ingresen a este mercado laboral generando bruscos movimientos en el ingreso promedio de este sector.

Sobre la base de esta evidencia, este trabajo propone que la presencia de la pequeña y mediana minería explica los impactos diferenciados que el sector minero genera sobre el ingreso regional, debido a una tecnología de producción más integrada con la actividad económica regional. Para testear estas hipótesis se propone una desagregación sectorial de las matrices insumo-producto regionales por tamaños de producción del sector minero. Esta descomposición permitirá el análisis de encadenamientos productivos y multiplicadores de producción e ingreso para cada subsector, indicadores que harían posible evaluar si la producción minera de pequeńa escala se encuentra más encadenada que la GMC.

\section{Metodología ${ }^{8}$}

Las tablas insumo-producto permiten tener una visión detallada de las estimaciones de flujos interindustriales en una economía. Desafortunadamente, el alto costo de representar todas las actividades productivas ha llevado a especificaciones a menudo insuficientemente detalladas. Esto es complicado cuando un sector productivo está compuesto por industrias de diferentes tamaños, con distintas combinaciones de tecnologías de producción. Si bien la agregación de tablas es uno de los temas que más ha sido desarrollado en la literatura insumo-producto, algunos autores han desarrollado metodologías de desagregación, proporcionando estimaciones de matrices de Leontief desagregadas.

Fei (1956) propone una sencilla forma de obtener una proxy de la matriz de Leontief a partir de las proporciones de participación de cada subsector en la producción bruta del sector agregado. La aproximación de Fei a la matriz de Leontief del modelo desagregado implica necesariamente la asignación de tecnologías de producción idénticas entre los subsectores, y entre los subsectores y el sector agregado, bloqueando con ello la posibilidad de diferentes tecnologías de producción al interior de una misma industria.

El detalle metodológico de la desagregación se encuentra disponible para quien lo solicite. 
A partir de la propuesta de Fei, y permitiendo al mismo tiempo la posibilidad de que existan diferencias entre la matriz de Leontief estimada y la matriz de Leontief original del modelo desagregado, Wolsky (1984) propone el modo de obtener la matriz de Leontief exacta del modelo desagregado o, en su defecto, los respectivos análisis de sensibilidad cuando no es posible contar con toda la información que el proceso exige. Específicamente, Wolsky propone una segunda matriz que corrige las diferencias entre la matriz propuesta por Fei y la verdadera matriz de Leontief del modelo desagregado, posibilitando que las diferencias entre ambas matrices sean causa de diferentes tecnologías de producción en cada subsector. La suma de ambas matrices dará como resultado la matriz de Leontief exacta del modelo desagregado.

Cuando la información necesaria no está disponible se pueden obtener intervalos que limitan la variación de los valores de la segunda matriz, y a partir de ello obtener múltiples estimaciones de la matriz de Leontief desagregada, generando todas las posibilidades exactas del modelo desagregado real. Este escenario da la opción de realizar un análisis de sensibilidad exacto, sobre todo los que se obtengan a partir de este modelo desagregado, obteniendo como resultado final intervalos de variación que incluirán todos los posibles valores de las variables parametrizadas desconocidas.

Sobre esta matriz de Leontief desagregada se calculan indicadores que permiten la comparación entre industrias y que son comúnmente denominados en la literatura como encadenamientos y multiplicadores?. San Cristóbal y Biezma (2006), por ejemplo, utilizan esta estrategia para corroborar a la minería como un sector clave en la Unión Europea, mediante el cálculo de encadenamientos hacia atrás y hacia adelante, mientras que Javorcik (2004) detecta, con el uso de encadenamientos hacia atrás, la existencia de externalidades positivas provenientes de la inversión extranjera directa para el caso de Lituania. En ambos casos el uso de encadenamientos ha facilitado el análisis de impacto sectorial.

La definición de encadenamientos y multiplicadores ocurre a partir de la identificación de dos efectos que la producción de cada sector provoca sobre el resto de la actividad productiva. El primero resulta de las compras efectuadas por el sector $j$, o específicamente, el efecto sobre los sectores proveedores de insumos para el sector $j$ derivado de aumentos en la demanda de este sector. El segundo efecto resulta de una mayor disponibilidad de oferta del sector $j$, o específicamente, el efecto sobre los sectores demandantes del producto del sector $j$ derivado de un aumento en la producción de este sector. Encadenamientos hacia atrás y encadenamientos hacia adelante son los términos utilizados para distinguir ambos efectos, que se obtienen de la siguiente forma ${ }^{10}$ :

$$
\begin{aligned}
& \overrightarrow{B^{\prime}}=\frac{\vec{\theta} \prime(I-A)^{-1}}{\vec{\theta} \prime(I-A)^{-1} \overrightarrow{\vec{e}}} \\
& \vec{F}=\frac{(I-A)^{-1} \overrightarrow{\vec{\theta}}}{\vec{\theta} \prime(I-A)^{-1} \vec{\theta}}
\end{aligned}
$$

9 En este caso, la comparación será en dos dimensiones: a nivel desagregado por escala de producción y a nivel regional.

10 La literatura sugiere normalizar siempre los encadenamientos utilizando el total de encadenamientos de la economía, para permitir la comparación entre dos economías diferentes. 
donde,

$\vec{B}^{\prime}=$ vector de encadenamientos normalizados hacia atrás de dimensión $(1 \times n)$

$\vec{F}=$ vector de encadenamientos normalizados hacia delante de dimensión $(n \times 1)$

$(I-A)^{-1}=$ matriz inversa de Leontief de dimensión $(n \times n)$

$\vec{e}=$ vector de unos de dimensión $(n \times 1)$

Por otro lado, los multiplicadores son estimaciones que describen el impacto que un potencial cambio exógeno de una variable generará sobre otras. Específicamente, los multiplicadores indican el efecto que un cambio en un componente agregado, como la demanda final o el empleo, generará sobre la economía en cuestión. Es posible dividir estos multiplicadores en dos tipos, tipo I y tipo II. Multiplicadores tipo I serán aquellos obtenidos utilizando un supuesto de economía abierta, en la cual todos los ingresos generados por conceptos de remuneraciones derivados de un cambio en la demanda final del sector $j$ no son gastados en la economía, mientras que los multiplicadores tipo ir asumen una economía cerrada en la que el total de los ingresos extras se gasta en la economía. En términos aplicados, los multiplicadores del tipo II utilizan una versión extendida de las matrices originales, que consiste en la inclusión de un vector columna correspondiente al consumo sectorial, y de un vector fila correspondiente a las remuneraciones por sector, esto es equivalente a asumir que las remuneraciones generadas en una economía son gastadas en ella.

Producto de la interdependencia que existe entre industrias, cada multiplicador se compone de tres efectos: efecto directo, indirecto e inducido. El efecto directo indica el requerimiento de producción necesario para satisfacer la variación en la demanda final del sector $j$; el efecto indirecto indica el requerimiento de producción de las industrias encargadas de suplir al sector $j$; y el efecto inducido resulta de un mayor nivel de empleo en la economía producto de estos múltiples requerimientos de insumos, lo que a su vez resulta nuevamente en un incremento del consumo. La forma de calcular los multiplicadores se presenta a continuación, para el caso de los dos multiplicadores más usados en la literatura: multiplicadores de la producción y del ingreso.

Multiplicador de la producción:

$$
\begin{array}{lll}
\text { Tipo I: } & O=\bar{e}^{\prime}(I-A)^{-1} \\
\text { Tipo II: } & \bar{O}=\bar{e}^{-1}(I-\bar{A})^{-1}
\end{array}
$$

Multiplicador del ingreso:

$$
\begin{array}{ll}
\text { Tipo I: } & H=\left[W *(\hat{X})^{-1}\right](I-A)^{-1} \\
\text { Tipo II: } & \bar{H}=\left[\bar{W} *(\hat{\bar{X}})^{-1}\right](I-\bar{A})^{-1}
\end{array}
$$

donde,

$O=$ vector de multiplicadores tipo I de dimensión $(1 \times n)$

$\bar{O}=$ vector de multiplicadores tipo II de dimensión $(1 \mathrm{x} n)$

$(I-A)^{-1}=$ matriz inversa de Leontief de dimensión $(n \times n)$ 
$(I-A)^{-1}=$ matriz inversa de Leontief extendida de dimensión $((n+1) \mathrm{x}(n+1))$

$\vec{e}=$ vector de unos de dimensión $(n \times 1)$

$\bar{e}=$ vector de unos de dimensión $((n+1) \times 1)$

$\hat{X}=$ vector de producción de dimensión $(1 \mathrm{x} n)$

$\hat{\bar{X}}=$ vector de producción extendida de dimensión $((n+1) \times 1)$ donde la entrada de la celda $(n+1)$ representa el total de la demanda de consumo en la economía.

$W=$ vector de remuneraciones de dimensión $(1 \mathrm{x} n)$

$\bar{W}=$ vector de remuneraciones extendidas de dimensión $(1 \mathrm{x}(n+1))$, donde la entrada de la celda $(n+1)$ representa la demanda de servicios del trabajo por el consumo en la economía.

\section{Estimación y resultados}

La metodología implica la realización de los siguientes pasos:

i. Desagregación del sector minería por tamaño de producción según Wolsky (1984); esto dará lugar a la generación de múltiples matrices exactas del modelo de Leontief desagregado que permitirán un análisis de sensibilidad.

ii. Obtención de encadenamientos y multiplicadores del producto y del ingreso, para la pequeña y mediana minería, y GMC, a partir de las múltiples opciones del modelo desagregado; el cálculo de multiplicadores a partir de sistemas abiertos y cerrados permitirá la obtención de intervalos para el verdadero valor del parámetro en cuestión.

iii. Estadísticos descriptivos de los encadenamientos y multiplicadores obtenidos, para cada tamańo de producción.

En cada etapa, los datos utilizados corresponden a las matrices regionales para Antofagasta y Atacama para 1996, publicadas por el INE (2004), con un nivel de agregación de doce sectores productivos.

La Tabla 3 presenta los resultados para encadenamientos y multiplicadores de la producción e ingreso para el sector minero en su especificación agregada, en ambas regiones. Los multiplicadores se han obtenido bajo los supuestos de economía abierta y economía cerrada. La tabla permite observar cómo la actividad minera alcanza mayores encadenamientos productivos, tanto hacia atrás como hacia adelante, en la región de Antofagasta. Esto significa que la actividad minera en Antofagasta genera mayores requerimientos de insumos (encadenamiento hacia atrás) y, al mismo tiempo, un mayor abastecimiento (encadenamiento hacia adelante), al resto de los sectores productivos de la región. Una explicación para ello, y sin rechazar la hipótesis de este trabajo, es que estos mayores encadenamientos se deban al volumen de producción de la región de Antofagasta.

El panorama cambia cuando se calculan multiplicadores bajo el supuesto de un sistema cerrado, en el que los ingresos adicionales son retenidos en su totalidad en la región donde se originan. Así, la Tabla 3 muestra cómo el multiplicador de la producción bajo esta modalidad es mayor en la región de Atacama. Es decir, el efecto que provocaría la retención total de los ingresos derivados de la extracción de 
cobre al interior de la región sería mayor en la actividad industrial de Atacama. Al mirar este multiplicador, se observa que a pesar de que el efecto directo sea mayor en Antofagasta, tanto los efectos indirectos como el efecto inducido, el que captura el efecto de la recirculación de ingresos en la economía, son mayores para la región de Atacama. Esta última idea es consistente con los resultados obtenidos para los multiplicadores del ingreso, para los cuales tanto en sistema abierto como cerrado, los mayores efectos también ocurren en Atacama.

TABLA $3 \mid$ Encadenamientos y multiplicadores del Sector Minería en presencia gradual de la producción de pequeña y mediana escala. Año 1996

\begin{tabular}{|l|c|c|}
\hline \multicolumn{1}{|c|}{ INDICADOR / REGIÓN } & ANTOFAGASTA & ATACAMA \\
\hline Encadenamientos Hacia Atrás & $1,121^{*}$ & 1.101 \\
\hline Encadenamientos Hacia Adelante & $1,008^{*}$ & 0,915 \\
\hline Multiplicador de la Producción Sistema Abierto & $1,435^{*}$ & 1.418 \\
\hline Efecto Directo & $1,326^{*}$ & 1.313 \\
\hline Efecto Indirecto & $0,110^{*}$ & 0,104 \\
\hline Multiplicador de la Producción Sistema Cerrado & 1.796 & $1,892^{*}$ \\
\hline Efecto Directo & $1,326^{*}$ & 1.313 \\
\hline Efecto Indirecto & 0,470 & $0,579^{*}$ \\
\hline Efecto Inducido & 0,360 & $0,475^{*}$ \\
\hline Multiplicador del Ingreso Sistema Abierto & 0,201 & $0,258^{*}$ \\
\hline Multiplicador del Ingreso Sistema Cerrado & 0,292 & $0,380^{*}$ \\
\hline
\end{tabular}

* Corresponde al Mayor valor entre Regiones

FUENTE ELABORACIÓN PROPIA A PARTIR DE DATOS DEL INE

\section{Análisis de sensibilidad}

El análisis de sensibilidad para la desagregación de las tablas insumo-producto se llevó a cabo a partir de la estructura de coeficientes técnicos regionales y de las participaciones en la producción regional de cada escala. Asumiendo que las tecnologías de producción se han mantenido constantes a lo largo del tiempo, las participaciones utilizadas corresponden al promedio de los años con información disponible, entre 2001 y 2008. A partir de 10.000 simulaciones de las matrices insumo-producto se calcularon encadenamientos y multiplicadores de la producción e ingresos para cada tamaño de producción. Lo siguiente es un análisis descriptivo de las distribuciones obtenidas.

Las Figuras 6 y 7 presentan las distribuciones para los encadenamientos hacia atrás y adelante para la GMC y la pequeña y mediana minería en cada región. Los estadísticos descriptivos para estos encadenamientos se presentan en la Tabla 4. A partir de la Figura 6 es posible ver cómo la distribución de encadenamientos hacia atrás para la pequeńa y mediana minería en ambas regiones se encuentra a la derecha de aquella obtenida para la GMC, sugiriendo que la pequeña minería está más encadenada que la GMC. Así, por cada dólar de aumento en la demanda de la pequeña y 
mediana minería, la demanda requerida al resto de los sectores productivos se incrementa en 2,58 dólares si esta se ubica en Antofagasta o en 1,23 dólares si se localiza en Atacama, mientras que la GMC solo logra incrementar la demanda de los otros sectores en aproximadamente 1 dólar, independientemente de su localización. La Figura 7, en cambio, es enfática en mostrar que los mayores encadenamientos hacia adelante en ambas regiones corresponden a la GMC, cuyos aportes varían entre 0,97 y 1,14 dólares, sea que se ubique en Atacama o en Antofagasta. Estos resultados son consistentes con lo obtenido previamente del análisis agregado. Un mayor encadenamiento hacia adelante en la GMC se entiende al considerar que, en su proceso extractivo, la gran minería no solo extrae cobre, sino que además logra extraer otros materiales que sirven de insumos a la industria manufacturera, por ejemplo en la fabricación de productos químicos. Se espera que este tipo de eventos ocurra en la minería de gran escala, debido a la tecnología de producción que poseen, y no así en la minería de pequeña escala o artesanal.

\section{FIGURA 6 Encadenamientos hacia atrás. Densidades por escala de producción y} región
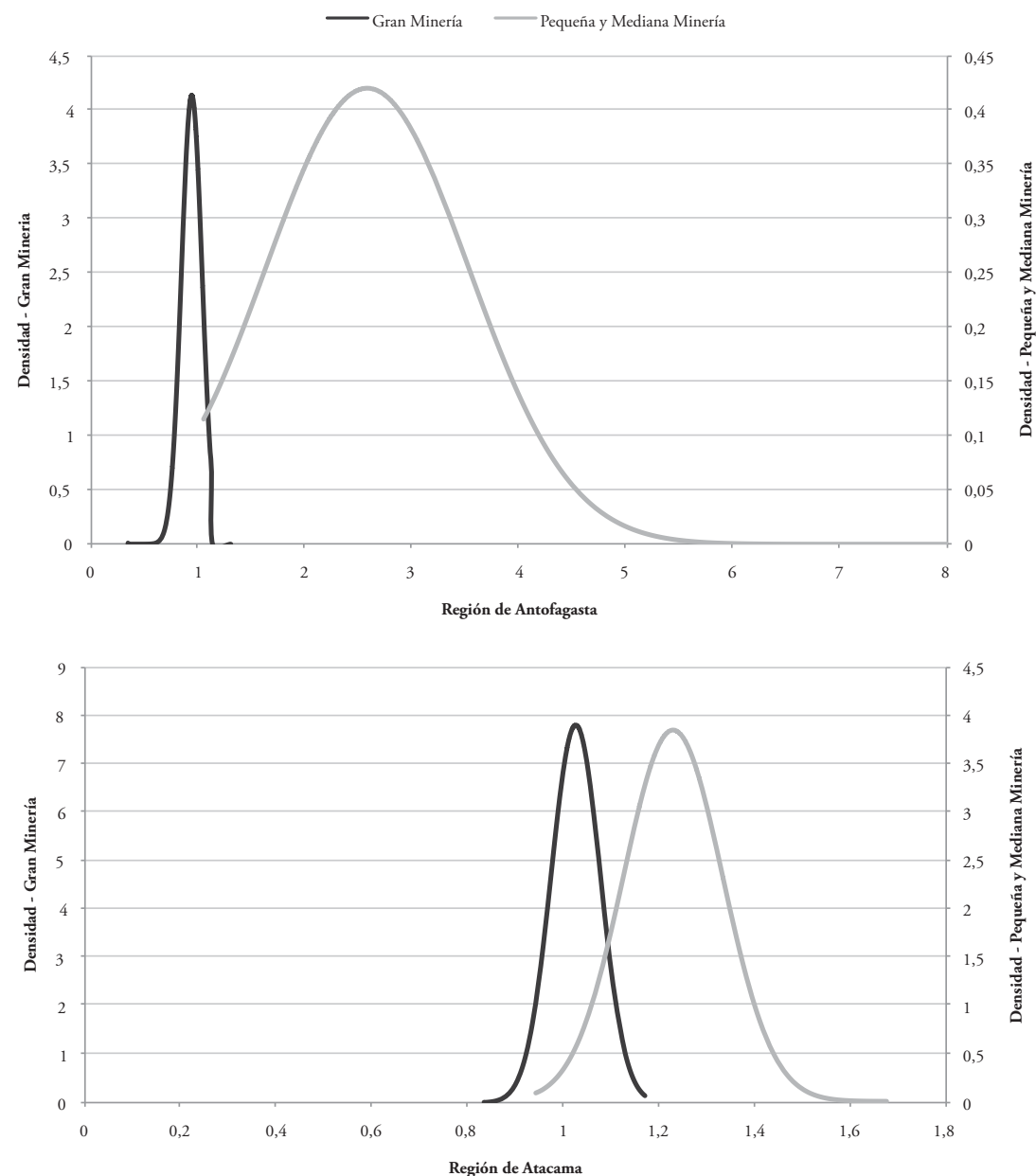

FUENTE ELABORACIÓN PROPIA 
El resultado de los encadenamientos productivos del modelo desagregado es consistente con la hipótesis de que la pequeña y mediana minería está más encadenada hacia atrás que la GMC. La implicación de este resultado es la menor integración vertical que caracteriza a este tamaño de producción, lo que genera mayores incrementos en la demanda del resto de los sectores productivos ante aumentos de la demanda en minería.

\section{FIGURA 7 Encadenamientos hacia adelante. Densidades por escala de producción y región}
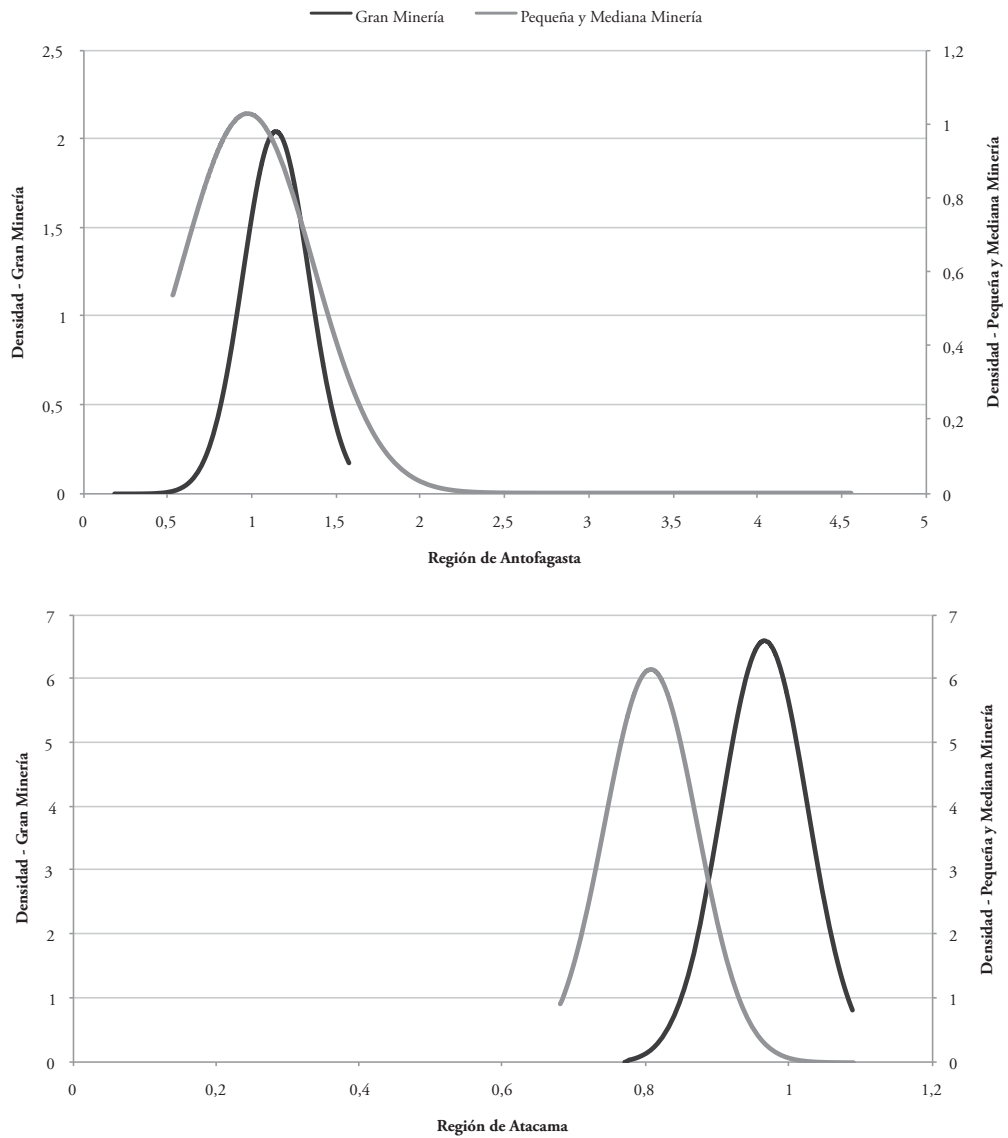

FUENTE ELABORACIÓN PROPIA

La Tabla 5 presenta los estadísticos descriptivos para multiplicadores de producto e ingreso. Específicamente, la tabla muestra la media y la desviación estándar en paréntesis para cada multiplicador a nivel regional por tamaño de empresa. En todos los casos los resultados son significativamente mayores, con $99 \%$ de confianza, para la pequeńa y mediana minería, independientemente de su localización espacial. En términos de producción, la mayor contribución la obtiene la pequeña y la mediana minería presentes en Antofagasta, resultado esperado considerando el nivel de encadenamientos hacia atrás de este sector en la región. Así, por ejemplo, cuando la demanda de la pequeña y mediana minería en Antofagasta se incrementa en 1 dólar, la producción de la totalidad de los sectores productivos de esta región 
aumenta en 4,16 dólares, casi 3 dólares más que el efecto generado por la producción de gran escala en la misma región. De este efecto en producción, 1,4 dólares tienen un efecto indirecto sobre la producción de toda la región, lo que significa que una vez descontado el primer efecto del incremento en la demanda de la pequeńa y mediana minería sobre la producción regional, que equivale a 2,74 dólares (efecto directo), 1,4 dólares extras son generados por el efecto multiplicador (efecto indirecto). Esto sugiere la posibilidad de que el tejido productivo de Antofagasta esté más interconectado que en Atacama, sobre todo al considerar que estos resultados son consistentes con un mayor efecto indirecto de la minería en Antofagasta en el modelo agregado (véase Tabla 3).

TABLA 4 Estadísticos descriptivos para encadenamientos productivos del modelo desagregado

\begin{tabular}{|l|c|c|c|}
\hline \multirow{2}{*}{ INDICADOR } & \multicolumn{1}{|c|}{ REGIÓN } & GMC & $\begin{array}{c}\text { PEQUEÑA Y } \\
\text { MEDIANA }\end{array}$ \\
\hline \multirow{3}{*}{ Encadenamiento Hacia Atrás } & \multirow{2}{*}{ Región de Antofagasta } & 0,93636 & $2,57892^{*}$ \\
\cline { 3 - 4 } & \multirow{2}{*}{ Región de Atacama } & $(0,09642)$ & $(0,94877)$ \\
\cline { 3 - 4 } & & $(0,05108)$ & $1,22871^{*}$ \\
\hline \multirow{3}{*}{ Encadenamiento Hacia Adelante } & \multirow{2}{*}{ Región de Antofagasta } & $1,13793^{*}$ & $0,10352)$ \\
\cline { 3 - 4 } & & $(0,19519)$ & $(0,38745)$ \\
\cline { 3 - 4 } & \multirow{2}{*}{ Región de Atacama } & $0,96578^{*}$ & 0,80676 \\
\cline { 3 - 4 } & & $(0,06043)$ & $(0,06484)$ \\
\hline
\end{tabular}

NOTA LOS ESTADÍSTICOS CORRESPONDEN A LA MEDIA Y DESVIACIÓN ESTÁNDAR (EN PARÉNTESIS) DERIVADOS DE IOOO SIMULACIONES. * COEFICIENTES SIGNIFICATIVOS AL I\% PARA EL TEST DE DIFERENCIAS DE MEDIAS ENTRE GMC Y PEQUEŃA Y MEDIANA MINERÍA VERSUS LA ALTERNATIVA DE MAYORES COEFICIENTES PARA LA PEQUEŃA Y MEDIANA MINERÍA A NIVEL REGIONAL.

FUENTE ELABORACIÓN PROPIA

Respecto del multiplicador del ingreso, los resultados del análisis de sensibilidad nuevamente indican mayores aportes a la pequeńa y mediana minería. Por cada incremento de 1 dólar en la demanda de este sector, 0,68 dólares extras son generados en Antofagasta por concepto de ingresos, mientras que en Atacama esta cifra corresponde a 0,3 dólares. Es importante mencionar que los resultados anteriores operan bajo el supuesto de una economía abierta, en donde todos los ingresos generados en la región se van de ella. Sin embargo, y acorde con lo anterior, la pequeña y mediana minería se caracterizan por poseer, en su mayoría, capitales locales a la región donde operan, con ello es de esperar que la mayoría de las ganancias generadas en estas empresas estén contabilizadas como ingresos que luego son retenidos en la región. De esta forma, un análisis más certero sería la consideración de intervalos de variación para el verdadero efecto a partir de la consideración de sistemas abiertos y cerrados.

De esta forma, la pequeña y mediana minería aparecen como la escala de producción que más impactos genera en términos de producción e ingresos por unidad 
producida, y de una manera independiente a la localización espacial de la actividad. A partir de los resultados, se espera que en términos de producción el impacto de la pequeña y mediana minería se encuentre entre 4,17 y 6,33 dólares, mientras que para la GMC este impacto fluctuará entre 1,4 y 1,74, aproximadamente, para el caso de Antofagasta. La situación es similar en Atacama, donde la pequeña y mediana minería obtienen un impacto en producción que oscila entre 1,58 y 2,29 dólares, mientras que el aporte de la GMC estaría entre 1,16 y 1,73 dólares, ante incrementos de 1 dólar en la demanda de cada sector. En términos de ingreso, el panorama no es muy diferente. Por cada dólar adicional en la demanda de la pequeña y mediana minería, se generan entre 0,68 y 1,16 dólares extras en la región de Antofagasta por concepto de ingresos, y entre 0,30 y 0,47 en la región de Atacama, incrementos significativamente mayores que aquellos generados por la GMC.

TABLA 5 Estadísticos descriptivos para multiplicadores del modelo desagregado

\begin{tabular}{|c|c|c|c|}
\hline INDICADOR & REGIÓN & GMC & $\begin{array}{c}\text { PEQUEÑA Y } \\
\text { MEDIANA }\end{array}$ \\
\hline \multirow{4}{*}{$\begin{array}{l}\text { Multiplicador de la Pro- } \\
\text { ducción Sistema Abierto }\end{array}$} & \multirow{2}{*}{ Región de Antofagasta } & 1,39678 & $4,16987^{*}$ \\
\hline & & $(0,14245)$ & $(4,85806)$ \\
\hline & \multirow{2}{*}{ Región de Atacama } & 1,1606 & $1,57623^{*}$ \\
\hline & & $(0,00039)$ & $(0,00069)$ \\
\hline \multirow{4}{*}{ Efecto Directo } & \multirow{2}{*}{ Región de Antofagasta } & 1,19206 & $2,74480^{*}$ \\
\hline & & $(0,0408)$ & $(0,43357)$ \\
\hline & \multirow{2}{*}{ Región de Atacama } & 1,22992 & $1,49175^{*}$ \\
\hline & & $(0,05848)$ & $(0,12483)$ \\
\hline \multirow{4}{*}{ Efecto Indirecto } & \multirow{2}{*}{ Región de Antofagasta } & 0,20473 & $1,42507^{*}$ \\
\hline & & $(0,14807)$ & $(4,74036)$ \\
\hline & \multirow{2}{*}{ Región de Atacama } & 0,10812 & $0,11328^{*}$ \\
\hline & & $(0,01965)$ & $(0,05014)$ \\
\hline \multirow{4}{*}{$\begin{array}{l}\text { Multiplicador de la Pro- } \\
\text { ducción Sistema Cerrado }\end{array}$} & \multirow{2}{*}{ Región de Antofagasta } & 1,74278 & $6,33325^{*}$ \\
\hline & & $(0,29374)$ & $(9,71491)$ \\
\hline & \multirow{2}{*}{ Región de Atacama } & 1,72582 & $2,28571^{*}$ \\
\hline & & $(0,13069)$ & $(0,31047)$ \\
\hline \multirow{4}{*}{ Efecto Directo } & \multirow{2}{*}{ Región de Antofagasta } & 1,19206 & $2,74480^{*}$ \\
\hline & & $(0,0408)$ & $(0,43357)$ \\
\hline & \multirow{2}{*}{ Región de Atacama } & 1,22993 & $1,49175^{*}$ \\
\hline & & $(0,05848)$ & $(0,12483)$ \\
\hline \multirow{4}{*}{ Efecto Indirecto } & \multirow{2}{*}{ Región de Antofagasta } & 0,55072 & $3,58845^{*}$ \\
\hline & & $(0,29814)$ & $(9,61883)$ \\
\hline & \multirow{2}{*}{ Región de Atacama } & 0,4959 & $0,79396^{*}$ \\
\hline & & $(0,10667)$ & $(0,25613)$ \\
\hline
\end{tabular}


(continuación)

\begin{tabular}{|c|c|c|c|}
\hline INDICADOR & REGIÓN & GMC & $\begin{array}{c}\text { PEQUEÑA Y } \\
\text { MEDIANA }\end{array}$ \\
\hline \multirow{4}{*}{ Efecto Inducido } & \multirow{2}{*}{ Región de Antofagasta } & 0,34599 & $2,16337^{*}$ \\
\hline & & $(0,15683)$ & $(5,0027)$ \\
\hline & \multirow{2}{*}{ Región de Atacama } & 0,38777 & $0,68068^{*}$ \\
\hline & & $(0,10199)$ & $(0,24103)$ \\
\hline \multirow{4}{*}{$\begin{array}{l}\text { Multiplicador del Ingreso } \\
\text { Sistema Abierto }\end{array}$} & \multirow{2}{*}{ Región de Antofagasta } & 0,20319 & $0,67762^{*}$ \\
\hline & & $(0,02455)$ & $(0,83221)$ \\
\hline & \multirow{2}{*}{ Región de Atacama } & 0,24212 & $0,29682^{*}$ \\
\hline & & $(0,01239)$ & $(0,02949)$ \\
\hline \multirow{4}{*}{$\begin{array}{l}\text { Multiplicador del Ingreso } \\
\text { Sistema Cerrado }\end{array}$} & \multirow{2}{*}{ Región de Antofagasta } & 0,28119 & $1,16157^{*}$ \\
\hline & & $(0,05482)$ & $(1,79819)$ \\
\hline & \multirow{2}{*}{ Región de Atacama } & 0,34113 & $0,47063^{*}$ \\
\hline & & $(0,03156)$ & $(0,07485)$ \\
\hline
\end{tabular}

NOTA LOS ESTADÍSTICOS CORRESPONDEN A LA MEDIA Y DESVIACIÓN ESTÁNDAR (EN PARÉNTESIS) DERIVADOS DE IOOO SIMULACIONES. ${ }^{*}$ COEFICIENTES SIGNIFICATIVOS AL I\% PARA EL TEST DE DIFERENCIAS DE MEDIAS ENTRE GMC Y PEQUEŃA Y MEDIANA MINERÍA VERSUS LA ALTERNATIVA DE MAYORES COEFICIENTES PARA LA PEQUEÑA Y MEDIANA MINERÍA A NIVEL REGIONAL.

FUENTE ELABORACIÓN PROPIA

El análisis de sensibilidad del modelo desagregado indica cómo la pequeña y mediana minería generan un mayor impacto tanto en la actividad productiva de la región donde se localizan como en los ingresos de sus habitantes. El hecho de que estos resultados serían aún mayores si todos los ingresos fueran retenidos en la región, permite sugerir que cuando la propiedad del capital es local, los efectos de la minería sobre el tejido productivo de la unidad espacial donde opera aumentan considerablemente, sobre todo para la pequeña y mediana minería. Esto porque tanto los trabajadores como los propietarios de las pequeńas y medianas empresas tienden a vivir en la localidad donde se desarrolla la explotación, a diferencia de los trabajadores de la GMC, que en parte importante conmutan desde otras regiones (Aroca \& Atienza, 2008; 2011), generando una diferencia en multiplicadores aún mayor ${ }^{11}$. En otras palabras, el impacto general de la GMC estaría más cercano al multiplicador del tipo I de economía abierta debido a la conmutación que realizan sus trabajadores, mientras que el impacto general de la pequeña y mediana minería estaría más cercano a los del tipo II o de economía cerrada, donde los ingresos de sus trabajadores se gastan efectivamente en las regiones en las cuales estas empresas se localizan.

Lo anterior significaría que no solo la tecnología de producción desempeña un papel fundamental en la determinación de los impactos de la minería, sino que, además, incide en dichos impactos la estructura de propiedad de su capital. En este punto es importante destacar que, con la evidencia obtenida desde el análisis de sensibilidad del modelo desagregado, este trabajo sugiere que mayores impactos del

11 Para un análisis similar sobre cómo la conmutación de trabajadores o la fuga de capitales de una región reducen los niveles de impactos económicos sobre una economía local, véase, por ejemplo, Ivanova y Rolfe (2011). 
sector minero en la producción y el ingreso regional se deben a diferentes tecnologías de producción en cada escala productiva. Sin embargo, y de forma adicional, es necesaria más investigación que permita descomponer de forma clara cuánto de la diferencia encontrada corresponde al tamaño de producción y cuánto, en cambio, se debe a otros factores, como - por ejemplo- la propiedad del capital de las empresas o la conmutación de sus trabajadores. Por otro lado, y como concluyen Karagiannis y Tzouvelekas (2010) para el caso de países de la Unión Europea, resultados positivos derivados de encadenamientos hacia atrás y hacia adelante debieran ser considerados en los análisis de política de forma paralela a la eficiencia en la utilización de recursos, conceptos que generalmente exhiben una relación inversa a nivel sectorial.

\section{Conclusiones}

A lo largo de este trabajo se revisa cómo la escala de producción de un sector productivo puede afectar las condiciones de desarrollo y bienestar de los habitantes de la unidad espacial en la que dicho sector se localiza. Específicamente se evalúa cómo la producción de menor escala afecta los niveles de ingreso de una economía regional.

Considerando el funcionamiento del sector minero en Chile, y su concentración espacial en las regiones de Antofagasta y Atacama, este trabajo plantea que la pequeńa y mediana minería generan impactos diferenciados en las dinámicas de ingreso regional por tonelada de cobre producida, debido a una tecnología de producción diferente a la que utiliza la gran minería. A partir de múltiples desagregaciones por tamaño de producción para el sector minería en las matrices insumo-producto disponibles para estas regiones, se obtuvieron encadenamientos y multiplicadores de la producción y del ingreso para la gran minería y la pequeńa y mediana minería del cobre.

El análisis de las distribuciones de estos indicadores sectoriales indica que la pequeña y mediana minería se encuentran más encadenadas hacia atrás que la GMC, independientemente de su localización espacial. Esto demuestra una tecnología de producción diferente para este tipo de empresas, que les permitiría una mayor conexión con el resto de sectores productivos regionales, a diferencia de lo que ocurre con la GMC. La razón más probable para ello sería una menor integración vertical de la pequeńa minería. Esta idea es consistente con la magnitud de las utilidades derivadas del proceso de explotación de la GMC. Mayores utilidades se traducen en un mayor valor agregado de las empresas de gran tamańo y, por lo tanto, una menor proporción de transacciones intermedias. Lo contrario ocurre en el caso de la pequeña y mediana minería. Unos mayores encadenamientos hacia atrás implican una menor proporción del valor agregado en la estructura productiva de este subsector, cuyas utilidades son mínimas en comparación con las obtenidas por la GMC. Esto corroboraría la hipótesis de una tecnología de producción diferente entre escalas de producción, con mayores requerimientos locales por tonelada de cobre producida, generados por la pequeńa y mediana minería. Es importante destacar en este punto que, debido a la explotación de carácter primario de este sector, las mayores ventajas de la pequeña y mediana minería provienen de los requerimientos de insumos que este sector demanda al resto de los sectores productivos, y 
no así de los encadenamientos hacia delante que este subsector pueda generar. Estas ventajas son aún mayores en Antofagasta, debido a la mayor industrialización que caracteriza a la pequeńa minería de esta región, a diferencia de lo que ocurre con Atacama, donde la pequeña minería es más bien de carácter artesanal.

Respecto de los multiplicadores y el efecto que incrementos en la demanda de la GMC y pequeńa y mediana minería generan sobre la producción y el ingreso regional, el panorama no es muy diferente. Al analizar estos valores bajo el supuesto de que todos los ingresos generados en la región son retenidos en su interior, los impactos que alcanzan la pequeña y mediana minería son significativamente mayores que aquellos generados por la GMC. Un mayor multiplicador de ingreso para la pequeña y mediana minería sugiere que la consideración simultánea de los salarios pagados y/o las utilidades obtenidas por esta escala de producción, además de los patrones de consumo de los hogares locales, genera un efecto multiplicador tal sobre la economía regional que supera al de la GMC. Al mismo tiempo, un mayor multiplicador de ingreso bajo la modalidad de un sistema cerrado sugiere que la propiedad local de las empresas generaría estímulos mayores sobre el ingreso regional. Ello porque las utilidades derivadas del proceso de explotación serían gastadas en la región donde son generadas, ya sea en forma de inversiones o salarios de sus dueños. Si bien esto no es suficiente para aceptar de manera concluyente la hipótesis de que unos mayores impactos de la pequeńa y mediana minería sobre el ingreso regional se deben a la propiedad local de su capital, este trabajo sugiere que tampoco es indicativo su descarte. Un análisis de causalidad que permita corroborar los efectos de una propiedad local versus una propiedad extranjera, además de una descomposición de cuánto de los impactos en ingreso regional se debe a las escalas de producción y a la propiedad del capital respectivamente, forman parte de los próximos trabajos en esta línea de investigación.

El análisis realizado en este trabajo comprueba la necesidad de atención sobre factores que parecieran ser clave en los incrementos del bienestar regional. Si elementos como la escala de producción condicionan efectivamente la función de producción de la minería, las estrategias de desarrollo a escala regional debieran considerar, desde su génesis, la forma de desarrollo del sector que domina la producción local, así como la cantidad de interconexiones que estos sectores generan con el resto de sectores productivos. De esta forma, elementos endógenos a la región podrían ayudar a mejorar el panorama industrial del sector dominante. Mayores incentivos a la formación de pequeños empresarios locales, y más atención a la fuga de capitales, son aspectos que - de acuerdo con este trabajo- necesitan ser considerados a la hora de delinear una estrategia de desarrollo regional que no desconozca las diferencias en composición y desarrollo del principal sector económico de la región. Al mismo tiempo, el análisis conjunto de estos resultados y de la política que a nivel país existe para las empresas de pequeńo tamańo en Chile, sugiere la necesidad de contar con planes de apoyo e incentivos para la formación de pequeńas y medianas empresas mineras que, de forma paralela a la actual política de fomento de la gran minería del cobre, potencien el desarrollo de este subsector, en virtud de su rentabilidad social y local, según ha sido aquí estudiada. 


\section{Referencias bibliográficas}

Argall, G. O. (1978). Future of small-scale mining - important for the future. Conference UNITAR. Nueva York, EE.uU.

Aroca, P. (2000). Impacto de la minería en la II Región. En P. Meller, Dilemas y debates en torno al cobre (pp. 79-100). Santiago: Dolmen Ediciones.

Aroca, P. (2001). Impacts and development in local economies based on mining: The case of the Chilean II Region. Resources Policy, 27(2), 119-134. http://dx.doi.org/10.1016/ S0301-4207(01)00013-7

Aroca, P. \& Atienza, M. (2008). La conmutación regional en Chile y su impacto en la Región de Antofagasta. EURE, 34(102), 97-120. http://dx.doi.org/10.4067/S025071612008000200006

Aroca, P. \& Atienza, M. (2011). Economic implications of long distance commuting in the Chilean mining industry. Resources Policy, 36(3), 196-203. http://dx.doi.org/10.1016/j. resourpol.2011.03.004

Banco Central de Chile. (2010). Base de Datos Estadísticos. Recuperado de Banco Central de Chile: www.bcentral.cl

Banks, G. (1993). Mining multinationals and developing countries: Theory and practice in Papua New Guinea. Applied Geography, 13(4), 313-327. http://dx.doi.org/10.1016/01436228(93)90035-Y

Capello, R. (2007). Regional Economics. Nueva York: Routledge.

Chinitz, B. (1961). Contrast in agglomeration: New York and Pittsburgh. The American Economic Review, 51(2), 279-289.

Comisión Chilena del Cobre. (2008). Anuario. Recuperado de Comisión Chilena del Cobre: www.cochilco.cl

Comisión Chilena del Cobre. (2010). Anuario. Recuperado de Comisión Chilena del Cobre: www.cochilco.cl

Fei, J. C. (1956). A fundamental theorem for the aggregation problem of input-output analysis. Econometrica, Journal of the Econometric Society, 24(4),400-412.

Ghose, M. K. \& Roy, S. (2007). Contribution of small-scale mining to employment, development and sustainability - An Indian scenario. Enviroment, Development and Sustainability, 9(3), 283-303. doi: 10.1007/s10668-006-9024-9

Gunton, T. (2003). Natural resources and regional development: An assessment of dependency and comparative advantage paradigms. Economic Geography, 79(1), 67-94. doi: 10.1111/j.1944-8287.2003.tb00202.x

Heim, C. E. (1983). Industrial organization and regional development in interwar Britain. Journal of Economic History, 43(4), 931-952. http://dx.doi.org/10.1017/ S0022050700030825

Hilson, G. M. (2002a). Small-scale mining and its socio-economic impact in developing countries. Natural Resources Forum, 26(1), 3-13. doi: 10.1111/1477-8947.00002

Hilson, G. M. (2002b). The Future of Small-scale Mining: Environmental and Socioeconomic Perspectives. Futures, 34, 863-872.

Instituto Nacional de Estadísticas (INE), Chile. (2004). Matrices insumo-producto regionales 1996. Santiago: Autor. 
Ivanova, G. \& Rolfe, J. (2011). Using input-output analysis to estimate the impact of a coal industry expansion on regional and local economies. Impact Assessment and Project Appraisal, 29(4), 277-288. http://dx.doi.org/10.3152/146155111X12959673795840.

Javorcik, B. S. (2004). Does foreign direct investment increase the productivity of domestic firms? In search of spillovers through backward linkages. The American Economic Review, 94(3), 605-627. doi: 10.1257/0002828041464605

Karagiannis, G. \& Tzouvelekas, V. (2010). Sectoral linkages and industrial efficiency: A dilemma or a requisition in identifying development priorities? The Annals of Regional Science, 45(1), 207-233. doi 10.1007/s00168-008-0280-5

Loveman, G. \& Sengenberger, W. (1991). The re-emergence of small-scale production: An international comparison. Small Business Economics, 3(1), 1-37.

Lufin, M. (2002). El caso del cluster minero de la Región de Antofagasta. Revista Ambiente y Desarrollo, 18(2-3-4), 106-111. En http://www.cipma.cl/web/200.75.6.169/ RAD/2002/2-3-4_Lufin.pdf

Rosenthal, S. S. \& Strange, W. C. (2003). Geography, industrial organization, and agglomeration. The Review of Economic and Statistics, 85(2), 377-393. doi: $10.1162 / 003465303765299882$

Rosenthal, S. S. \& Strange, W. C. (2010). Small establishments/Big effects: Agglomeration, industrial organization and entrepreneurship. En E. L. Glaeser, Agglomeration Economics (pp. 277-302). Chicago: The University of Chicago Press.

Salas, A. (2009a). La mediana minería en Chile. En Empresa Nacional de Minería (Enami), Chile minero: Enami en la historia de la pequeña y mediana minería chilena (pp. 221221). Santiago: Ocho Libros Editores.

Salas, A. (2009b). Planes de cierre en la pequeña y mediana minería. Presentación en XII Seminario de Medio Ambiente: Planes de Cierre de Faenas Mineras. Organizado por el Instituto de Ingenieros de Minas de Chile (ІІмсн), La Serena, 8 de mayo de 2009. En http://www.iimch.cl/documentos/sonami_albertosalas.pdf

San Cristóbal, J. R. \& Biezma, M. V. (2006). The mining industry in the European Union: Analysis of inter-industry linkages using input-output analysis. Resources Policy, 31(1), 1-6. http://dx.doi.org/10.1016/j.resourpol.2006.03.004

Saxenian, A. (1994). Regional Advantage: culture and competition in Silicon Valley and Route 128. Cambrigde, MA: Harvard University Press.

Shen, L. \& Gunson, A. J. (2006). The role of artisanal and small-scale mining in China's economy. Journal of Cleaner Production, 14(3), 427-435. http://dx.doi.org/10.1016/j. jclepro.2004.08.006

Spilimbergo, A. (2002). Copper and the Chilean economy, 1960-98. Journal of Economic Policy Reform, 5(2), 115-126. doi: 10.1080/13841280214094

Spilling, O. R. (1998). On the re-emergence of small scale production: The Norwegian case in international comparison. Small Business Economics, 10(4), 401-417.

Wolsky, A. M. (1984). Disaggregating input-output models. The Review of Economics and Statistics, 66(2), 283-291.

Yelpaala, K. \& Ali, S. H. (2005). Multiple scales of diamond mining in Akwatia, Ghana: Addressing environmental and human development impact. Resources Policy, 30(3), 145-155. http://dx.doi.org/10.1016/j.resourpol.2005.08.001 